\title{
Effects of $R f-1, R f-3$ and $R f-6(t)$ Genes on Fertility Restoration in Rice (Oryza sativa L.) with WA- and BT-type Cytoplasmic Male Sterility
}

\author{
Yuichi Tada \\ School of Bionics, Tokyo University of Technology, 1404-1 Katakura, Hachioji, Tokyo 192-0982, Japan
}

Genes involved in the fertility restoration $(R f)$ of wild-abortive (WA)-type cytoplasmic male sterility (CMS) in rice have not been clearly identified, because of the inconsistency of their number and map position. Although the $R f-1$ genes, which restore Chinsurah Boro (BT)-type CMS, have been recently isolated, their effects on WA-type CMS are still unclear. In the present study, near-isogenic lines (NILs) of japonica rice, differing at $R f$ loci, were developed using marker-assisted selection (MAS) to determine the effect of each $R f$ gene on WA- or BT-cytoplasm in a japonica background. The NILs were crossed with a japonica CMS line, then the pollen and seed fertility of the hybrid plants was analyzed. The $R f-1$ locus consisting of two $R f$ genes from IR36 was sufficient to restore fertility in the hybrids with BT-type CMS but conferred very little fertility to those with WA-type CMS. $R f-3$ on chromosome 1 , a weak $R f$ gene, and $R f-6(t)$ on chromosome 10 were unable to confer seed fertility to plants with BT-type or WA-type CMS. Both $R f$ genes, however, could accelate the development of abortive pollen in the case of WA-type CMS and their effect was additive. Crossing of CMS rice with chromosome segment substitution lines carrying $R f$ loci from IR24 resulted in similar pollen development and seed fertility.

Key Words: cytoplasmic male sterility (CMS), fertility restorer, wild-abortive (WA), Chinsurah Boro (BT), near-isogenic lines.

\section{Introduction}

Cytoplasmic male sterility (CMS) is a maternally inherited trait caused by the incompatibility between the nucleus and cytoplasm, resulting in the inability to produce fertile pollen. This phenomenon is widely observed in higher plants. Male fertility in plants with CMS is restored by nuclear fertility-restorer $(R f)$ genes.

$R f$ genes have been cloned in corn (Cui et al. 1996), petunia (Bentolila et al. 2002), kosena radish (Koizuka et al. 2003) and radish (Brown et al. 2003). In rice (Oryza sativa L.), $R f-1$, a gene that restores Chinsurah Boro- or BT-type CMS, has also been cloned (Akagi et al. 2004, Komori et al. 2004, Kazama and Toriyama 2003). Wang et al. (2006) demonstrated that the locus consists of a cluster of duplicated genes. BT-type CMS is used for seed production of japonica hybrid varieties (Fujimura et al. 1996).

In indica rice, hybrid varieties have been developed using wild abortive (WA)-type CMS. Although fertility can be restored by the dominant restorer genes, reports on the number, positions and effects of these $R f$ genes are inconsistent. The presence of one $R f$ gene has been reported in some studies (Shen et al. 1996) and of two in others (Raj and Virmani 1988, Bharaj et al. 1991, 1995, Teng and Shen 1994, Tan et

Communicated by H. Kato

Received January 12, 2007. Accepted June 19, 2007.

*Corresponding author (e-mail: tadayui@bs.teu.ac.jp) al. 1998, Jing et al. 2001). Raj and Virmani (1986) reported that an appropriate combination of two of eight restorer genes fully restored fertility in $F_{1}$ hybrids with WA-type CMS. Furthermore, the locations of such genes have been reported as follows: one on chromosome 1 (Zhang et al. 1997), two on chromosomes 7 and 10 (Bharaj et al. 1995), two on chromosomes 1 and 10 (Yao et al. 1997), two on chromosome 10 (Tan et al. 1998, Jing et al. 2001) and four quantitative trait loci on chromosomes 2, 3, 4 and 5 (Zhu et al. 1996). These findings suggest that indica rice exhibits genetic diversity for $R f$ genes. It was reported that the restoration of fertility in IR36 was governed by two independent and dominant genes, one of which appeared to be stronger than the other (Raj and Virmani 1988). Bharaj et al. (1995) reported that the stronger gene was located on chromosome 7 and the weaker one on chromosome 10 in IR36. Zhang et al. (1997) reported that $R f-3$ in IR36 and IR24 was located on chromosome 1.

In the present study, near-isogenic lines (NILs) of japonica carrying $R f-1, R f-3$ and/or $R f-6(t)$ loci from IR36 were developed using marker-assisted selection (MAS). NILs were used to study the effects of the $R f$ genes on fertility restoration in rice with CMS, regardless of the interaction with the unidentified $R f$ genes in indica. Chromosome segment substitution lines (CSSLs) (Kubo et al. 2002) were also used as male lines to study the effects of $R f$ loci from a different nuclear background. In the CSSLs, chromosome segments of Asominori (japonica) were substituted with those 
from IR24 corresponding to $R f$ loci. Japonica lines with CMS possessing WA and BT cytoplasm were also developed by MAS and conventional backcrossing, respectively. The effects of the $R f$ genes on both WA- and BT-type CMS were studied by crossing the CMS-rice with the NILs or CSSLs.

\section{Materials and Methods}

\section{Plant materials}

Although the $R f-1$ locus consists of two genes, Rfla and $R f 1 b$ (Wang et al. 2006), the genes were tightly linked and co-segregated in the NILs, CSSLs and $\mathrm{F}_{1}$ plants used in the present study. Therefore, we treated these $R f-1$ genes as a single locus, $R f-1$, in this report, unless otherwise mentioned.

The CMS line IR68902A was used as a donor of WA cytoplasm. Kinuhikari (japonica variety, $r f-1 / r f-1, r f-3 / r f-3$, $r f-6(t) / r f-6(t))$ was used as a donor of japonica nuclear material. BT-type CMS lines of Kinuhikari have been derived by backcrossing Kinuhikari to Taichung 65A (Chu et al. 1972). Two indica rice varieties, IR36 $(R f-1 / R f-1, R f-3 / R f-3, R f$ $6(t) / R f-6(t))$ and IR24 (Rf-1/Rf-1, Rf-3/Rf-3, Rf-6(t)/Rf-6(t)), were used as donors of $R f$ genes because their fertility restoration on WA-type CMS had been well-documented. IR36 was used to develop NILs of Kinuhikari differing at the $R f$ loci. CSSLs AIS1 and AIS72 (Kubo et al. 2002), in which chromosome segments of Asominori (japonica) are substituted with those of IR24 (indica), were provided by Dr. Yoshimura (Kyusyu university). AIS1 includes $R f-3$, while AIS72 includes both $R f-1$ and $R f-6(t)$. Rice plants were cultivated in pots $9 \mathrm{~cm}$ in diameter in a greenhouse or incubation room $\left(25^{\circ} \mathrm{C}, 12 \mathrm{~h}\right.$ light $)$.

\section{Marker-assisted selection (MAS)}

Plant DNA was extracted from a $5 \times 5 \mathrm{~mm}$ piece of young seedling tissue according to the method of Edwards et al. (1991). One micro-liter of diluted DNA in $100 \mu \mathrm{l}$ of TE buffer (10 mM Tris-HC1, $1 \mathrm{mM}$ EDTA, pH 8.0) was used as a template for the polymerase chain reaction (PCR) with ExTaq polymerase (Takarabio, Ohtsu, Japan) in a total volume of $10 \mu \mathrm{l}$. The composition of the PCR solution followed the supplier recommendations. The PCR conditions for simple sequence repeat (SSR) markers consisted of 40 cycles of $10 \mathrm{~s}$ at $94^{\circ} \mathrm{C}, 30 \mathrm{~s}$ at $55^{\circ} \mathrm{C}$ and $30 \mathrm{~s}$ at $72^{\circ} \mathrm{C}$. SSR markers (Akagi et al. 1996, Panaud et al. 1996, Chen et al. 1997, Temnykh et al. 2000, 2001, McCouch et al. 2002) showing polymorphism between IR68902A and Kinuhikari or Kinuhikari and IR36 were selected and used for MAS (Fig. 1). The single nucleotide polymorphic marker 01-45 was used to detect the $R f 1 b$ sequence according to the method of Wang et al. (2006). The $R f-1 A$ (Rfla) gene was detected by PCR using the specific primers Rf1-3F (5'-tgatgatcgaggaggaggta-3') and Rf1-4R ( $5^{\prime}$-aagagtattgtaggtaatgac-3'), which were designated according to the reported $R f-1 A$ sequence (Komori et al. 2004). Size of the specific PCR product was $1009 \mathrm{bp}$.

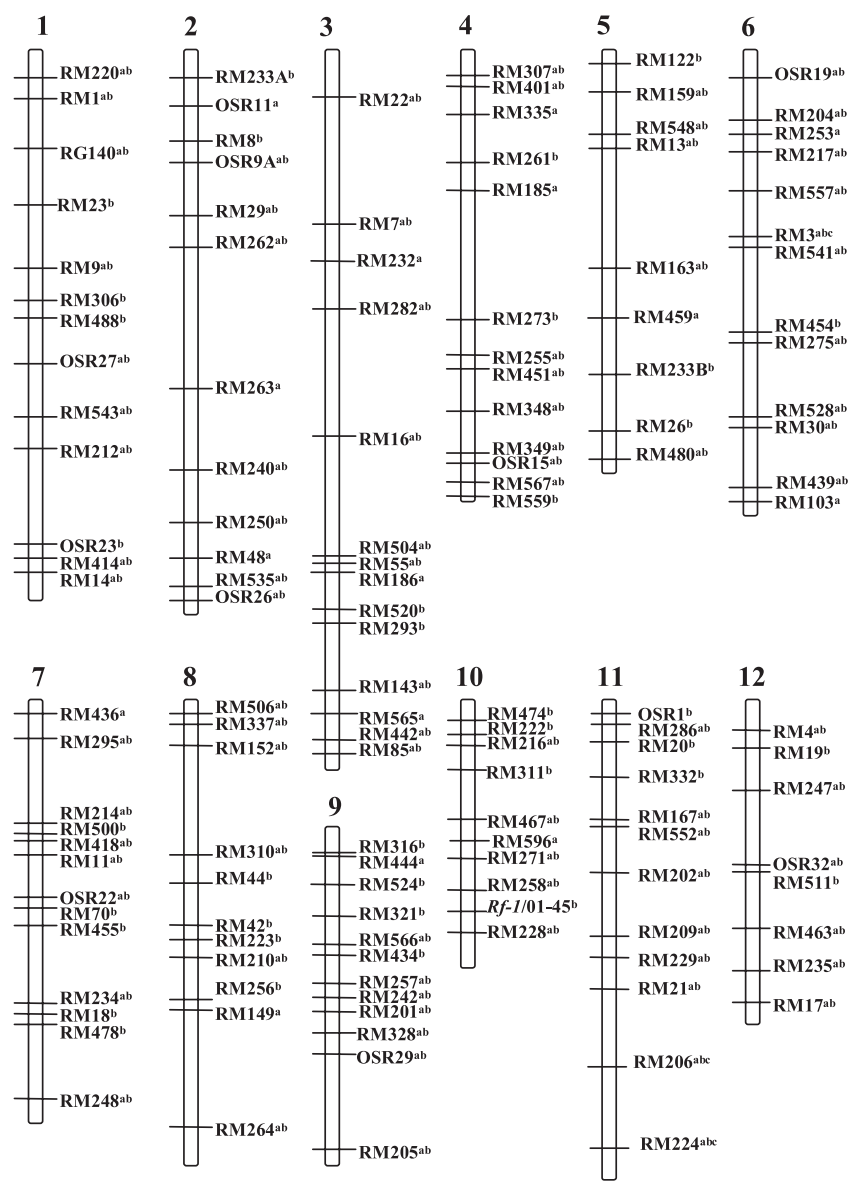

Fig. 1. Chromosomal positions of the DNA markers used to develop the WA-type cytoplasmic male sterile (CMS) line and nearisogenic lines (NILs). ${ }^{\mathrm{a}}$ and ${ }^{\mathrm{b}}$ indicate the markers used to develop the WA-type CMS line and NILs of Kinuhikari, respectively.

Cleaved amplified polymorphic sequences (CAPS) marker RG140 (Nguyen et al. 1998) was used to select NILs with $R f-3$. Due to the use of the CAPS marker RG140, the PCR products were digested with a restriction enzyme, EcoRI, before being subjected to electrophoresis. The PCR conditions for $R f-1 A$ and RG140 consisted of 35 cycles of $10 \mathrm{~s}$ at $94^{\circ} \mathrm{C}, 30 \mathrm{~s}$ at $60^{\circ} \mathrm{C}$ and $30 \mathrm{~s}$ at $72^{\circ} \mathrm{C}$. PCR products were separated by electrophoresis on 3\% MetaPhor agarose gels (Takarabio, Ohtsu, Japan).

\section{Production and genotyping of $F_{1}$ plants}

KinuhikariA (BT-type CMS) and KinuhikariWA (WAtype CMS) were crossed with the NILs developed in this study and with the CSSLs AIS1 and AIS72. Since the NIL KinuhikariRf6(t) carrying $R f-6(t)$ and the NIL KinuhikariRf1Rf3Rf6(t) carrying $R f-1, R f-3$ and $R f-6(t)$ were heterozygous at each $R f$ locus, the genotype of the $\mathrm{F}_{1}$ plants was determined using the corresponding DNA markers.

\section{Determination of pollen and seed fertility}

Three to ten anthers were randomly sampled from each 
plant to determine the pollen fertility at the flowering stage. Pollen grains were stained in an $\mathrm{I}_{2}$-KI solution containing $0.18 \%$ iodine and $1 \%$ potassium iodide and observed under a light microscope. Pollen fertility was classified into five classes $0,<5,30,50$ and $>80 \%$ by the stainability, shape and size of pollen grains. Two major panicles from each plant were covered with a paper bag before flowering and the seed fertility was determined by counting the seeds that set after maturation.

\section{Results}

\section{Development of japonica CMS lines and NILs}

A CMS line carrying WA-type CMS was isolated by backcrossing Kinuhikari to IR68902A four times and selecting the seedlings that showed the maximum number of Kinuhikari-type bands using SSR markers covering the whole genome (Fig. 1). This CMS line, in which all the SSR markers showed Kinuhikari-type bands, was designated as KinuhikariWA. A CMS line with BT-type CMS isolated by backcrossing Kinuhikari to Taichung 65A eight times was designated as KinuhikariA.

The NILs of Kinuhikari carrying the $R f-1$ or $R f-3$ gene were developed by repeatedly backcrossing Kinuhikari to IR36/Kinuhikari. The $\mathrm{BC}_{1} \mathrm{~F}_{1}$ plants were screened for the production of $R f-1 A$-specific fragments or non-cleaved products of RG140 when digested with EcoRI (Nguyen et al. 1998). Then a genome-wide scan was carried out to determine the maximum number of Kinuhikari-type bands using 101 SSR markers in the plants, homozygous or heterozygous at the locus (Fig. 1 and Fig. 2A). The selected plants were backcrossed to Kinuhikari and re-screened for $R f-1 A$ specific products or RG140, followed by the SSR markers for three generations. From the $\mathrm{BC}_{3} \mathrm{~F}_{2}$ generation, NILs of Kinuhikari with $R f-1 A$ and $R f-3$ were developed and designated as KinuhikariRf1 and KinuhikariRf3 (Fig. 2A), respectively. Then, the presence of $R f 1 b$ in KinuhikariRf1 was detected using the 01-45 marker. KinuhikariRf1 may also include $R f-4$ because these two loci may be allelic (Jing et al. 2001) or tightly linked (Tan et al. 1998). Another NIL with
$R f-6(t)$, KinuhikariRf6(t), was developed from the progeny of Kinuhikari/IR36 by a similar procedure. On chromosome 10 , the presence of one quantitative trait locus (QTL) or $R f$ $6(t)$ gene which confers partial fertility in plants with WAtype CMS was reported (Tan et al. 1998, Jing et al. 2001). The QTL was mapped between the restriction fragment length polymorphism (RFLP) markers RG257 and S11014 (Tan et al. 1998). Rf-6(t) was also identified in this region (Jing et al. 2001). Because this region closely corresponded to that between the SSR markers RM311 and RM258 (McCouch et al. 2000, 2002), both these and two additional markers between them, RM467 and RM271, were used to develop the NIL, KinuhikariRf6(t) (Fig. 2A), carrying $R f$ $6(t)$. Similarly, another NIL KinuhikariRf1Rf3Rf6(t), with heterozygous $R f-1, R f-3$ and $R f-6(t)$ loci, (Fig. 2A) was developed by MAS. The presence of $R f 1 b$ in KinuhikariRf1Rf3Rf6(t) was also confirmed using the 01-45 marker.

CSSLs AIS1 and AIS72 were developed by Kubo et al. (2002), in which chromosome segments of Asominori (japonica) were substituted with those of IR24. Their graphical genotypes are shown in Fig. 2B. AIS1 includes $R f-3$, while AIS 72 includes both $R f-1$ and $R f-6(t)$, from IR24.

\section{Pollen fertility of BT-type CMS crossed with NILs and CSSLS}

KinuhikariA (BT-type CMS) was crossed with the NILs and CSSLs and the $R f$ genotype of the resulting CMS/ NILs $F_{1}$ plants was determined by gene diagnosis using the above DNA markers. Since the NILs KinuhikariRf6(t) and KinuhikariRf1Rf3Rf6(t) were heterozygous at each $R f$ locus, segregation of each $R f$ gene was examined using the corresponding DNA markers, as shown in Table 1.

The pollen grain viability assay with $\mathrm{I}_{2}-\mathrm{KI}$ revealed that the pollen grains of KinuhikariA were faintly stained and round in shape (Fig. 3A), as reported by Chu et al. (1972).

KinuhikariA was crossed with each NIL to examine the pollen and seed fertility of the $F_{1}$ plants. Pollen fertility of the $\mathrm{F}_{1}$ plants is also summarized in Table 1. KinuhikariA/ KinuhikariRf1 $\mathrm{F}_{1}$ plants exhibited the genotype $R f-1 / r f-1$,

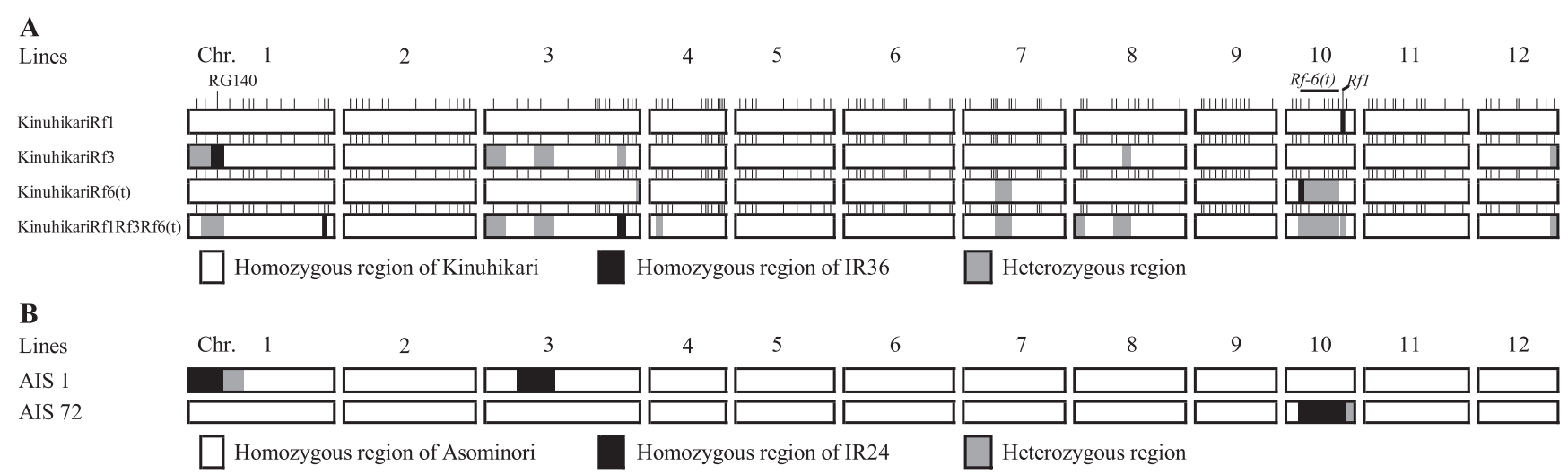

Fig. 2. Graphical genotypes of (A) the near isogenic lines (NILs) and (B) chromosome segment substitution lines (CSSLs). (A) NILs of Kinuhikari differing at $R f$ loci. (B) CSSLs of Asominori. Modified from Kubo et al. (2002). 
$r f-3 / r f-3, r f-6(t) / r f-6(t)$ and the fertile pollen with $R f-1$ and sterile pollen with $r f-1$ segregated at a ratio of $1: 1$ (Fig. 3B), in line with the fact that BT-type CMS showed gametophytic fertility restoration. The KinuhikariA/ KinuhikariRf1Rf3Rf6(t) $\mathrm{F}_{1}$ plants $(R f-1 / r f-1, R f-3 / r f-3, r f$ $6(t) / r f-6(t))$, KinuhikariA/KinuhikariRf1Rf3Rf6(t) $\mathrm{F}_{1}$ plants $(R f-1 / r f-1, \quad r f-3 / r f-3, \quad R f-6(t) / r f-6(t))$ and KinuhikariA/ KinuhikariRf1Rf3Rf6(t) $\mathrm{F}_{1}$ plants $(R f-1 / r f-1, R f-3 / r f-3, R f$ $\sigma(t) / r f-6(t))$ also had a fertile and sterile pollen at a ratio of $1: 1$ (Fig. 3E, F, H). The KinuhikariA/KinuhikariRf3 $\mathrm{F}_{1}$ plants $(r f-1 / r f-1, R f-3 / r f-3, r f-6(t) / r f-6(t))$, KinuhikariA/ KinuhikariRf6(t) $\mathrm{F}_{1}$ plants $(r f-1 / r f-1, r f-3 / r f-3, R f-6(t) / r f-$ $\sigma(t))$ and KinuhikariA/KinuhikariRf1Rf3Rf6(t) $\mathrm{F}_{1}$ plants $(r f-1 / r f-1, R f-3 / r f-3, R f-6(t) / r f-6(t))$ produced faintly stained pollen grains (Fig. 3C, D, G). Although it was difficult to determine precisely the pollen fertility because of its spectrum ranging from faintly-stained to darkly-stained grains, the proportion of darkly stained pollen grains of normal size was estimated to be less than 5\% (Table 1).
Similarly, the pollen fertility of KinuhikariA pollinated with the CSSLs was studied (Table 1). The KinuhikariA/ AIS72 $\mathrm{F}_{1}$ plants $(R f-1 / r f-1, r f-3 / r f-3, R f-6(t) / r f-6(t))$ had a fertile and sterile pollen at a ratio of $1: 1$. While the KinuhikariA/AIS1 $\mathrm{F}_{1}$ plants $(r f-1 / r f-1, R f-3 / r f-3, r f-6(t) /$ $r f-6(t))$ produced only faintly stained sterile pollen grains.

Seed fertility of BT-type CMS crossed with NILs and CSSLs Seed fertility of KinuhikariA crossed with the NILs and CSSLs is also summarized in Table 1. KinuhikariA maintained by crossing with Kinuhikari was stable and sterile. The $\mathrm{F}_{1}$ plants from KinuhikariA/KinuhikariRf1 (Rf-1/rf-1, $r f-3 / r f-3, r f-6(t) / r f-6(t))$ with a pollen fertility of about $50 \%$ showed a seed fertility in the range of $71.4-84.2 \%$, indicating near normal levels of fertility. Seed fertility of two KinuhikariA/KinuhikariRf1Rf3Rf6(t) $\mathrm{F}_{1}$ plants $(R f-1 / r f-1$, $R f-3 / r f-3, r f-6(t) / r f-6(t))$ was in the range of $52.9-68.6 \%$. The seed fertility of the KinuhikariA/KinuhikariRf1Rf3Rf6(t) $\mathrm{F}_{1}$ plants $(R f-1 / r f-1, r f-3 / r f-3, R f-6(t) / r f-6(t))$ ranged from 80.0

Table 1. Pollen and seed fertility of BT-type CMS crossed with near-isogenic lines (NILs) and chromosome segment substitution lines (CSSLs)

\begin{tabular}{|c|c|c|c|c|c|c|c|c|}
\hline \multirow{2}{*}{ Female } & \multirow{2}{*}{ Male } & \multirow{2}{*}{$R f$ loci } & \multirow{2}{*}{$\begin{array}{l}\text { Class of pollen } \\
\text { fertility }^{1)}(\%)\end{array}$} & \multicolumn{5}{|c|}{ Seed fertility (\%) } \\
\hline & & & & $\# 1$ & $\# 2$ & \#3 & $\# 4$ & $\# 5$ \\
\hline \multirow[t]{8}{*}{ KinuhikariA } & Kinuhikari & - & 0 & 0 & 0 & 0 & 0 & 0 \\
\hline & KinuhikariRf1 & $R f-1$ & 50 & 77.1 & 71.4 & 72.2 & 84.2 & \\
\hline & KinuhikariRf3 & $R f-3$ & $<5$ & 0 & 0 & 0 & 0 & 0 \\
\hline & KinuhikariRf6(t) & $R f-6(t)$ & $<5$ & 0 & 0 & 0 & 0 & 0 \\
\hline & KinuhikariRf1Rf3Rf6(t) & $R f-1, R f-3$ & 50 & 52.9 & 56.4 & 68.6 & & \\
\hline & KinuhikariRf1Rf3Rf6(t) & $R f-1, R f-6(t)$ & 50 & 89.0 & 90.9 & 80.0 & & \\
\hline & KinuhikariRf1Rf3Rf6(t) & $R f 3, R f-6(t)$ & $<5$ & 0 & 0 & 0 & 0 & \\
\hline & KinuhikariRf1Rf3Rf6(t) & $R f-1, R f 3, R f-6(t)$ & 50 & 83.2 & 90.5 & & & \\
\hline \multirow[t]{2}{*}{ KinuhikariA } & AIS1 & $R f-3$ & 0 & 0 & 0 & 0 & 0 & \\
\hline & AIS72 & $R f-1, R f-6(t)$ & 50 & 91.1 & 84.6 & 78.0 & 97.4 & \\
\hline
\end{tabular}

1) $F_{1}$ plants with the same genotypes showed a similar pollen fertility.

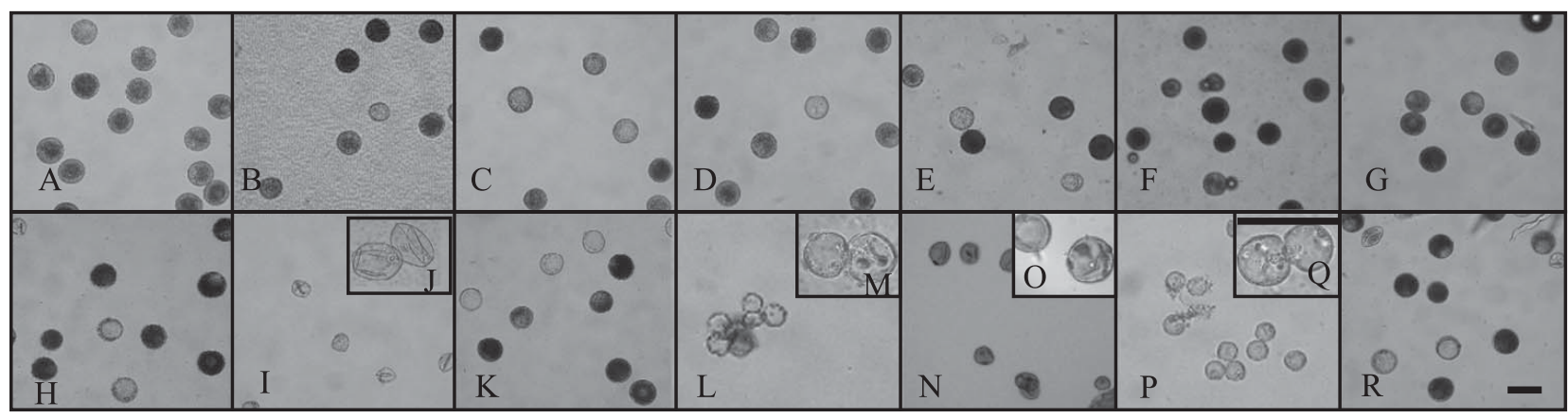

Fig. 3. $\mathrm{I}_{2}-\mathrm{KI}$ stainability of pollen grains of the CMS lines and $\mathrm{F}_{1}$ plants. (A) KinuhikariA; (B) KinuhikariA/KinuhikariRf1 (Rf-1/rf-1, $r f-3 /$ $r f-3, r f-6(t) / r f-6(t))$; (C) KinuhikariA/KinuhikariRf3 $(r f-1 / r f-1, R f-3 / r f-3, r f-6(t) / r f-6(t))$; (D) KinuhikariA/KinuhikariRf6(t) $(r f-1 / r f-$ 1, $r f-3 / r f-3, R f-6(t) / r f-6(t))$; (E) KinuhikariA/KinuhikariRf1Rf3Rf6(t) $(R f-1 / r f-1, R f-3 / r f-3, r f-6(t) / r f-6(t))$; (F) KinuhikariA/ KinuhikariRf1Rf3Rf6(t) $(R f-1 / r f-1, r f-3 / r f-3, R f-6(t) / r f-6(t))$; (G) KinuhikariA/KinuhikariRf1Rf3Rf6(t) $(r f-1 / r f-1, R f-3 / r f-3$, $R f-6(t) / r f-6(t))$; (H) KinuhikariA/KinuhikariRf1Rf3Rf6(t) $(R f-1 / r f-1, R f-3 / r f-3, R f-6(t) / r f-6(t))$; (I) and (J) KinuhikariWA; (K) KinuhikariWA/KinuhikariRf1 $(R f-1 / r f-1, r f-3 / r f-3, r f-6(t) / r f-6(t))$; (L) and (M) KinuhikariWA/KinuhikariRf3 (rf-1/rf-1, Rf-3/ $r f-3, r f-6(t) / r f-6(t))$; (N) and (O) KinuhikariWA/KinuhikariRf6(t) $(r f-1 / r f-1, r f-3 / r f-3, R f-6(t) / r f-6(t))$; (P) and (Q) KinuhikariWA/ KinuhikariRf1Rf3Rf6(t) $(r f-1 / r f-1, R f-3 / r f-3, R f-6(t) / r f-6(t))$; and (R) KinuhikariWA/KinuhikariRf1Rf3Rf6(t) $(R f-1 / r f-1, R f-3 /$ $r f-3, R f-6(t) / r f-6(t))$. Horizontal bars indicate $50 \mu \mathrm{m}$. 
to $90.9 \%$. The seed fertility of the KinuhikariA/ KinuhikariRf1RF3Rf6(t) $\mathrm{F}_{1}$ plants $(\mathrm{R} f-1 / r f-1, R f-3 / r f-3, R f-$ $6(t) / r f-6(t))$, which harbor three $R f$ genes, was in the range of 83.2 to $90.5 \%$. The $F_{1}$ plants of KinuhikariA crossed with NILs which harbored $R f-3, R f-6(t)$ or both could not set seed despite the fact that $<5 \%$ of their pollen grains was darkly stained by $\mathrm{I}_{2} \mathrm{KI}$. These results indicated that the $R f-1$ gene plays a major role in the fertility restoration of BT-type CMS.

The seed fertility of KinuhikariA crossed with the CSSLs carrying $R f$ loci from IR24 is summarized in Table 1. The KinuhikariA/AIS72 $\mathrm{F}_{1}$ plants $(R f-1 / r f-1, r f-3 / r f-3, R f$ $6(t) / r f-6(t))$ showed a seed fertility of $78.0-97.4 \%$, indicating near normal levels of fertility. The KinuhikariA/AIS $1 \mathrm{~F}_{1}$ plants $(r f-1 / r f-1, R f-3 / r f-3, r f-6(t) / r f-6(t))$ were sterile. These results corresponded to the seed fertility of CMS/NILs carrying $R f$ loci from IR36.

\section{Pollen fertility of WA-type CMS crossed with NILs and} CSSLS

KinuhikariWA (WA-type CMS) was crossed with the NILs and CSSLs. The $R f$ genotype of the CMS/NILs $\mathrm{F}_{1}$ plants was determined by gene diagnosis using DNA markers and is shown in Table 2.

In the parental line KinuhikariWA, the pollen grains could not be stained and were shrunken (Fig. 3I, J), which is typical of indica rice with WA-type CMS (Shen et al. 1996). The pollen fertility of the KinuhikariWA/NIL and KinuhikariWA/CSSL $\mathrm{F}_{1}$ plants is also summarized in Table 2.

The KinuhikariWA/KinuhikariRf1 $\mathrm{F}_{1}$ plants dipplayed the genotype $R f-1 / r f-1, r f-3 / r f-3, r f-6(t) / r f-6(t)$. Pollen grains stained with $\mathrm{I}_{2}$-KI showed a spectrum ranging, from unstained vacuolated to darkly-stained grains (Fig. 3K). Their size also varied from normal to rather larger. Darkly-stained but larger pollen grains were considered to be sterile because rice plants producing pollens grains of in such an abnormal size never set seed in the present study. Although it was difficult to determine precisely pollen fertility, the proportion of darkly stained pollen grains of normal size was less than 5\% (Table 2). Although the KinuhikariWA/KinuhikariRf3 $\mathrm{F}_{1}$ plants $(r f-1 / r f-1, R f-3 / r f-3, r f-6(t) / r f-6(t))$ mostly pro- duced unstained and shrunken pollen grains; the appearance of the pollen grains was clearly distinguishable from that of the pollen grains from the parental line KinuhikariWA. About $20 \%$ of the pollen grains in the $F_{1}$ plants were round and vacuolated (Fig. $3 \mathrm{~L}, \mathrm{M}$ ), indicating that the stage of development was advanced, compared with that of KinuhikariWA. Similar pollen grains were observed in the KinuhikariWA/KinuhikariRf6(t) $\mathrm{F}_{1}$ plants (rf-1/rf-1, $r f-3 /$ $r f-3, R f-6(t) / r f-6(t))$ (Fig. 3N, O) and the KinuhikariWA/ KinuhikariRF1Rf3Rf6(t) $\mathrm{F}_{1}$ plants $(r f-1 / r f-1, R f-3 / r f-3$, $R f-6(t) / r f-6(t))$ (Fig. 3P, Q). KinuhikariWA/KinuhikariRf1Rf3Rf6(t) $\mathrm{F}_{1}$ plants $(R f-1 / r f-1, R f-3 / r f-3, R f-6(t) / r f-6(t))$ produced pollen grains showing a spectrum ranging from unstained vacuolated to darkly-stained grains. However, the proportion of darkly stained pollen grains of normal size was less than 5\% (Fig. 3R and Table 2).

Although the KinuhikariWA/AIS72 $\mathrm{F}_{1}$ plants $(R f-1 / r f$ $1, r f-3 / r f-3, R f-6(t) / r f-6(t))$ mostly produced shrunken, or round and vacuolated pollen grains; a few grains were darkly-stained (data not shown). The KinuhikariWA/AIS1 $\mathrm{F}_{1}$ plants $(r f-1 / r f-1, R f-3 / r f-3, r f-6(t) / r f-6(t))$ produced mostly unstained and shrunken pollen grains, while a few grains were round and vacuolated (data not shown).

\section{Seed fertility of WA-type CMS crossed with NILs and CSSLS}

The seed fertility of KinuhikariWA crossed with the NILs and CSSLs is also summarized in Table2. KinuhikariWA was stable and sterile. The seed fertility of two KinuhikariWA/KinuhikariRf1 $\mathrm{F}_{1}$ plants $(R f-1 / r f-1, r f-3 / r f-3$, $r f-6(t) / r f-6(t))$ ranged from 2.0 to $6.0 \%$. The $\mathrm{F}_{1}$ plants of KinuhikariWA harboring either $R f-3$ or $R f-6(t)$, or both of them were completely sterile. However, the seed fertility of the KinuhikariWA/KinuhikariRf1Rf3Rf6(t) $\mathrm{F}_{1}$ plants $(R f-1 / r f-1, R f-3 / r f-3, R f-6(t) / r f-6(t))$ was in the range of 17.4 and $8.3 \%$. These results indicated that the $R f-1$ locus could partially restore the fertility of WA-type CMS and that $R f-3$ or $R f-6(t)$ exerted an additive effect on fertility restoration when combined with the $R f-1$ locus.

The seed fertility of KinuhikariWA/CSSLs $\mathrm{F}_{1}$ plants is summarized in Table 2. The seed fertility of the KinuhikariWA/AIS72 $\mathrm{F}_{1}$ plants $(R f-1 / r f-1, r f-3 / r f-3, R f-6(t) /$

Table 2. Pollen and seed fertility of WA-type CMS crossed with near-isogenic lines (NILs) and chromosome segment substitution lines (CSSLs)

\begin{tabular}{|c|c|c|c|c|c|c|c|c|}
\hline \multirow{2}{*}{ Female } & \multirow{2}{*}{ Male } & \multirow{2}{*}{ Rf loci } & \multirow{2}{*}{$\begin{array}{l}\text { Class of pollen } \\
\text { fertility }^{1)}(\%)\end{array}$} & \multicolumn{5}{|c|}{ Seed fertility (\%) } \\
\hline & & & & $\# 1$ & $\# 2$ & $\# 3$ & $\# 4$ & $\# 5$ \\
\hline \multirow[t]{6}{*}{ KinuhikariWA } & Kinuhikari & - & 0 & 0 & 0 & 0 & 0 & 0 \\
\hline & KinuhikariRf1 & $R f-1$ & $<5$ & 4.7 & 6.0 & 2.0 & 4.8 & 0 \\
\hline & KinuhikariRf3 & $R f-3$ & 0 & 0 & 0 & 0 & 0 & 0 \\
\hline & KinuhikariRf6(t) & $R f-6(t)$ & 0 & 0 & 0 & 0 & 0 & \\
\hline & KinuhikariRf1Rf3Rf6(t) & $R f 3, R f-6(t)$ & 0 & 0 & 0 & 0 & 0 & \\
\hline & KinuhikariRf1Rf3Rf6(t) & $R f-1, R f 3, R f-6(t)$ & $<5$ & 17.4 & 8.3 & & & \\
\hline \multirow[t]{2}{*}{ KinuhikariWA } & AIS1 & $R f-3$ & 0 & 0 & 0 & 0 & 0 & \\
\hline & AIS72 & $R f-1, R f-6(t)$ & $<5$ & 1.6 & 0 & 0 & & \\
\hline
\end{tabular}

1) $F_{1}$ plants with the same genotypes showed a similar pollen fertility. 
$r f-6(t))$ was in the range of $0-1.6 \%$, while that of the KinuhikariWA/AIS1 $\mathrm{F}_{1}$ plants $(r f-1 / r f-1, R f-3 / r f-3, r f-6(t) /$ $r f-6(t))$ was $0 \%$. These results corresponded to the seed fertility of CMS/NILs carrying $R f$ loci from IR36.

\section{Discussion}

Genetic studies on $R f$ genes using indica variety are complicated due to the inconsistency of the number and map position of the $R f$ genes involved (Shen et al. 1996, Raj and Virmani 1986, 1988, Bharaj et al. 1991, 1995, Teng and Shen 1994, Zhu et al. 1996, Yao et al. 1997, Zhang et al. 1997, Tan et al. 1998, Jing et al. 2001). I suggested that this phenomenon was associated with the indica genetic background which may contain unidentified genes that influence the pollen fertility. To confirm this assumption, I introduced the $R f S$ and CMS into the japonica variety and examined the effects of the $R f-1, R f-3$ and $R f-6(t)$ genes on fertility in BTand WA-type CMS.

It was reported that, in BT-type CMS, microspores failed to develop after release from tetrads and that almost all the microspores remained mononucleate ( $\mathrm{Chu}$ et al. 1972). In WA-type CMS, microspores failed to develop earlier after release from tetrads and the microspores formed was small, shrunken and could not be stained with $\mathrm{I}_{2}$-KI because of the lack of starch grains. I observed that the $R f-1$ gene was more or less effective on both cytoplasm types: the $R f-1$ derived from IR36 restored pollen fertility in a gametophytic manner in BT-type CMS (Table 1), and partially restored pollen fertility in WA-type CMS (Table 2). These results are consistent with those obtained in previous studies using indica and japonica varieties (Young and Virmani 1984, Raj and Virmani 1988, Bharaj et al. 1991, 1995, Teng and Shen 1994, Yao et al. 1997, Akagi et al. 2004, Komori et al. 2004, Wang et al. 2006). At present, it is remains to be determined whether the partial restoration of pollen fertility in WA-type CMS is a pleiotropic effect of $R f l a$ or $R f 1 b$ (Wang et al. 2006), or is due to the clustering of multiple $R f$ genes of the $R f-1$ locus. Nucleotide sequencing of the $R f-1$ locus revealed that it consists of multiple PPR protein genes, a gene family to which most of the plant $R f$ genes belong (Bentolila et al. 2002, Koizuka et al. 2003, Brown et al. 2003). Further studies should be carried out to identify the genes responsible for the fertility restoration of WA-type CMS of the $R f-1$ locus.

On the other hand, the effect of the $R f-3$ and $R f-6(t)$ genes is puzzling: they did not exert any effect on BT-type CMS. Also, neither the $R f-3$ nor $R f-6(t)$ gene was able to confer seed fertility to rice with WA-type CMS. It should, however, be noted that the appearance of the pollen grains was clearly different from that of the pollen grains of the parental line with WA-type CMS (Fig. 3L, M), presumably because the developmental stage of the pollen was advanced. In the presence of $R f-1, R f-3$ or $R f-6(t)$ improved seed fertility in rice with WA-type CMS (Table 2). Taken together, it was concluded that the presence of $R f-3$ and $R f-6(t)$ is not sufficient to restore pollen fertility but contributes to the to development of the pollen grains. These results contradict those previously reported, suggesting that $R f-3$ and/or $R f$ $6(t)$ play a major role in the restoration of WA-type CMS (Yao et al. 1997, Zhang et al. 1997, Tan et al. 1998, Jing et al. 2001). It should be considered that the previous studies had been conducted in indica genetic background, in which the $R f$ genes may occur. Unintended combination of such $R f$ genes in a genetic background may result in an apparent major role of $R f-3$ and $R f-6(t)$. In several reports, the presence of such genes in an indica genetic background was suggested (Raj and Siddiqi 1984, Raj and Virmani 1988, Yao et al. 1997, Zhuang et al. 2001). In this context, my approach using NILs in a japonica genetic background may enable to identify precisely the effects of the $R f$ genes. This approach could facilitate the genetic analysis of $R f$ genes in rice, including their molecular cloning.

\section{Acknowledgements}

I thank Dr. Khush, Dr. Motomura and Dr. Yoshimura for providing IR68902A, Taichung 65A and chromosome segment substitution lines, respectively.

\section{Literature Cited}

Akagi,H., Y.Yokozeki, A.Inagaki and T.Fujimura (1996) Microsatellite DNA markers for rice chromosomes. Theor. Appl. Genet. 94: 61-67.

Akagi, H., A. Nakamura, Y. Yokozeki-Misono, A. Inagaki, H. Takahashi, K.Mori and T.Fujimura (2004) Positional cloning of the rice $R f-1$ gene, a restorer of BT-type cytoplasmic male sterility that encodes a mitochondria-targeting PPR protein. Theor. Appl. Genet. 108: 1449-1457.

Bentolila, S., A.A.Alfonso and M.R.Hanson (2002) A pentatricopeptide repeat-containing gene restores fertility to cytoplasmic male-sterile plants. Proc. Natl. Acad. Sci. USA 99: 1088710892.

Bharaj,T.S., S.S.Bains, G.S.Sidhu and M.R.Gangneja (1991) Genetics of fertility restoration of 'wild abortive' cytoplasmic male sterility in rice, Oryza sativa L. Euphytica 56: 199-203.

Bharaj,T.S., S.S.Virmani and G.S.Khush (1995) Chromosomal location of fertility restoring genes for 'wild abortive' cytoplasmic male sterility using primary trisomics in rice. Euphytica 83: 169-173.

Brown, G.G., N.Formanava, H.Jin, R.Wargachuk, C.Dendy, P.Patil, M.Laforest, J.Zhang, W.Y.Cheung and B.S.Landry (2003) The radish $R f o$ restorer gene of Ogura cytoplasmic male sterility encodes a protein with multiple pentatricopeptide repeats. Plant J. 35: 262-272.

Chen,X., S.Temnykh, Y.Xu, Y.G.Cho and S.R.McCouch (1997) Development of microsatellite framework map providing genome-wide coverage in rice (Oryza sativa L.). Theor. Appl. Genet. 95: 553-567.

Chu,Y.E., Y.S.Li, C.Shinjyo and H.W.Li (1972) Hybrid rice breeding III. Cyto-histological investigation of pollen degeneration in anther of cytoplasmic male-sterile plants. Jpn. J. Genet. 47: 179-183.

Cui,X., R.P.Wise and P.S.Schnable (1996) The rf2 nuclear restorer 
gene of male-sterile T-cytoplasm maize. Science 272: 1334 1336.

Edwards, K., C.Johnstone and C.Thompson (1991) A simple and rapid method for the preparation of plant genomic DNA for PCR analysis. Nuc. Acid. Res. 19: 1349.

Fujimura,T., H.Akagi, M.Oka, A.Nakamura and R.Sawada (1996) Establishment of a rice protoplast culture and application of an asymmetric protoplast fusion technique to hybrid rice breeding. Plant Tissue Cult. Lett. 13: 243-247.

Jing, R., X.Li, P.Yi and Y.Zhu (2001) Mapping fertility-restoring genes of rice WA cytoplasmic male sterility using SSLP markers. Bot. Bull. Acad. Sci. 42: 167-171.

Kazama, T. and K. Toriyama (2003) A pentatricopeptide repeatcontaining gene that promotes the processing of aberrant atp6 RNA of cytoplasmic male-sterile rice. FEBS Lett. 544: 99102.

Koizuka,N., R.Imai, H.Fujimoto, T.Hayakawa, Y.Kimura, J.KohonoMurase, T.Sakai, S.Kawasaki and J.Imamura (2003) Genetic characterization of a pentatricopeptide repeat protein gene, orf687, that restores fertility in the cytoplasmic male-sterile Kosena radish. Plant J. 34: 407-415.

Komori,T., S.Ohta, N.Murai, Y.Takakura, Y.Kuraya , S. Suzuki, Y.Hiei, H.Imaseki and N.Nitta (2004) Map-based cloning of a fertility restorer gene, $R f-1$, in rice (Oryza sativa L.). Plant J. 37: 315-325.

Kubo, T., Y.Aida, K. Nakamura, H.Tsunematsu, K. Doi and A. Yoshimura (2002) Reciprocal chromosome segment substitution series derived from japonica and indica cross of rice (Oryza sativa L.) Breed. Sci. 52: 319-325.

McCouch, S.R., S.Temnykh, A.Lukashova, J.Coburn, G.DeClerck, S.Cartinhour, S. Harrington, M. Thomson, E. Septiningsih, M.Semon, P.Moncada and J.Li (2000) Microsatellite markers in rice: abundance, diversity, and applications. In "Rice Genetics IV Proc $4^{\text {th }}$ Int. Rice. Genet. Symp." Khush, G.S. and D. Brar (eds.), IRRI, Manila. p. 117-135.

McCouch,S.R., L.Teytelman, Y.Xu, K.B.Lobos, K.Clare, M.Walton, B.Fu, R.Maghirang, Z.Li, Y.Xing, Q.Zhang, I.Kono, M.Yano, R.Fjellstorm, G.DeClerck, D.Schneider, S.Cartinhour, D.Ware and L.Stein (2002) Development and mapping of 2240 new SSR markers for rice (Oryza sativa L.). DNA Res. 9: 199-207.

Nguyen, T.L., G. Zhang, G. Magpantay, S.S. Virmani, N. Huang, D.S.Brar, G.S.Khush and Z.K.Li (1998) PCR-based DNA markers for the WA-CMS fertility restoring gene $R f-3$ in rice. Rice Genet. Newsl. 15: 156-158.

Panaud,O., X.Chen and S.R.McCouch (1996) Development of microsatellite markers and characterization of simple sequence length polymorphism (SSLP) in rice (Oryza sativa L.). Mol. Gen. Genet. 252: 597-607.

Raj,K.G. and E.A.Siddiqi (1984) Genetics of fertility restoration and biochemical basis of male sterility-fertility restoration system in rice. Rice Genet. Newsl. 1: 103-104.

Raj,K.G. and S.S.Virmani (1986) Allelism test for restorer genes of six promising IR restorer lines. Rice Genet. Newsl. 3: 85-86.

Raj,K.G. and S.S.Virmani (1988) Genetics of fertility restoration of 'WA' type cytoplasmic male sterility in rice. Crop Sci. 28: 787-792.

Shen, Y., Q.Cai, M.Gao and X.Wang (1996) Isolation and genetic characterization of a fertility-restoring revertant induced from cytoplasmic male sterile rice. Euphytica 90: 17-23.

Tan, X.L., A.Vanavichit, S.Amornsilpa and S.Trangoonrung (1998) Genetic analysis of rice CMS-WA fertility restoration based on QTL mapping. Theor. Appl. Genet. 96: 994-999.

Temnykh, S., W.D. Park， N. Ayers， S. Cartinhour， N. Hauck， L. Lipovich, Y.G.Cho, T.Ishii and S.R.McCouch (2000) Mapping and genome organization of microsatellite sequences in rice (Oryza sativa L.). Theor. Appl. Genet. 100: 697-712.

Temnykh,S., G.DeClerck, A.Lukashova, L.Lipovich, S.Cartinhour and S.R.McCouch (2001) Computational and experimental analysis of microsatellites in (Oryza sativa L.): Frequency, length variation, transposon associations, and genetic marker potential. Genome Res. 11: 1441-1452.

Teng,L.-S. and Z.-T.Shen (1994) Inheritance of fertility restoration for cytoplasmic male sterility in rice. Rice Genet. Newsl. 11: 9597.

Wang,Z., Y.Zou, X.Li, Q.Zhang, L.Chen, H.Wu, D.Su, Y.Chen, J.Guo, D.Luo, Y.Long, Y.Zhong and Y.-G.Liu (2006) Cytoplasmic male sterility of rice with boro II cytoplasm is caused by a cytotoxic peptide and is restored by two related PPR motif genes via distinct modes of mRNA silencing. Plant Cell 18: 678-687.

Yao,F.Y., C.G.Xu, S.B.Yu, J.X.Li, Y.J.Gao, X.H.Li and Q.Zhang (1997) Mapping and genetic analysis of two fertility restorer loci in the wild-abortive cytoplasmic male sterility system of rice (Oryza sativa L.). Euphytica 98: 183-187.

Young,J.B. and S.S.Virmani (1984) Inheritance of fertility restoration in a rice cross. Rice Genet. Newsl. 1: 102-103.

Zhang, G., T.S.Bharaj, Y.Lu, S.S.Virmani and N.Huang (1997) Mapping of the Rf-3 nuclear fertility-restoring gene for WA cytoplasmic male sterility in rice using RAPD and RFLP markers. Theor. Appl. Genet. 94: 27-33.

Zhu,L., C.Lu, P.Li, L.Shen, Y.Xu, P.He and Y.Chen (1996) Using doubled haploid populations of rice for quantitative trait locus mapping, In "Rice Genetics III Proc $3^{\text {rd }}$ Int. Rice. Genet. Symp.” Khush, G.S. (ed.), IRRI, Manila, p. 631-635.

Zhuang,J.Y., Y.Y.Fan, J.L.Wu, Z.M.Rao, Y.W.Xia and K.L.Zheng (2001) Mapping genes for rice CMS-WA fertility restoration. Yi Chuan Xue Bao 28: 129-134 (in Chinese). 